\title{
Die 7. WTO-Ministerkonferenz in Genf 2009
}

\author{
Von Nicolas Sonder, Frankfurt*
}

\section{Vorgeschichte}

Vom 30.11.2009 bis zum 02.12.2009 fand unter den gewöhnlich lauten Protesten und Demonstrationen seit vier Jahren wieder eine WTO-Ministerkonferenz statt. Manch einer wird das im Trubel der unzähligen Zusammenkünfte anderer supranationaler Organisationen und Staaten zur Krisenbewältigung vielleicht kaum noch bemerkt haben. Doch tatsächlich trafen sich in Genf die Wirtschafts- und Handelsminister der insgesamt 153 Mitgliedstaaten, um mit festen Zielen vor Augen und unter dem Motto „Die WTO, das multilaterale Handelssystem und das gegenwärtige globale ökonomische Umfeld“ über Herausforderungen, Probleme sowie deren Lösungen für die Weltwirtschaft zu diskutieren. Konkret sollte es dabei zum einen auch um die Folgen der Finanz- und Wirtschaftskrise von 2008 für die Weltwirtschaft gehen. Man hatte sich zum Ziel gesetzt, gerade jetzt mehr Transparenz zu schaffen und die Mitgliedstaaten von protektionistischen Maßnahmen abzuhalten. ${ }^{1}$ Zum anderen sollte die Doha-Runde von 2001, welche für einen verbesserten Marktzugang, den Abbau von Zöllen sowie von Exportsubventionen (vor allem im Bereich der landwirtschaftlichen Produkte) stand, auf der Agenda zu finden sein. ${ }^{2}$ Weil diese Runde bekannterweise zum Stillstand gebracht wurde, sollte von der Konferenz nun endlich ,ein starkes Signal ausgehen“. Pascal Lamy sprach in der Öffentlichkeit bisweilen gar davon, dass es in absehbarer Zeit zum Abschluss der Doha-Liberalisierungsrunde kommen müsse. ${ }^{3}$ Hingegen traten schon im Vorfeld der Konferenz Bedenken zu Tage, etwa hinsichtlich der Bedeutung der WTO in der Krise. Ferner stand der Abbau von Zöllen nicht auf der offiziellen Agenda. Fraglich erschien auch, ob die USA ihre Agrarsubventionen abbauen würden, ohne dabei einen merklich verbesserten Marktzugang zu Entwicklungsländern zu bekommen. ${ }^{4}$

* Nicolas Sonder, Dr. iur., Wissenschaftlicher Mitarbeiter an der Professur für deutsches und internationales Privat- und Wirtschaftsrecht von Prof. Dr. Axel Halfmeier an der Frankfurt School of Finance \& Management. E-Mail: Nicolas.Sonder@gmail.com

1 Fontana, WTO und Protektionismus: Die Lamy-Initiative, Die Volkswirtschaft 12-2009, S. 65 und 66.

2 Vgl. zu diesen Inhalten der Doha-Runde Krajewski, Wirtschaftsvölkerrecht (2006), § 2, Rdn. 199ff.

3 Insofern zum Ausblick auf die Konferenz Euro am Sonntag v. 28.11.2009, S. 5.

Mönch, WTO: Stillstand bei Handelsgesprächen, Agrarzeitung v. 27.11.2009, S. 3. 


\section{Ergebnisse der Konferenz}

Entscheidend sind freilich die Ergebnisse, welche die Konferenz dann hervorbrachte. Es versteht sich von selbst, dass zunächst seitens des Generaldirektors sowie der Vorsitzenden der Arbeitausschüsse abschließend festgestellt wurde, dass die Mitgliedstaaten gemeinsame Anstrengungen und politische Energie zur Beendigung der Doha-Runde an den Tag legten. Dabei will man von nun an noch mehr auf die Bedürfnisse der Entwicklungsländer und der am wenigst entwickelten Staaten („LDC“), etwa durch Verbesserung des Markzugangs, Steigerung der Entwicklungshilfe und dem Abschluss neuer bilateraler und multilateraler Handelsabkommen, eingehen. Verstärkt wurde dieser Optimismus durch die Entscheidung, schon 2010 neue Konsultationen für eine nächste Ministerkonferenz im Jahr 2011 durchzuführen. ${ }^{5}$. Hinsichtlich der Bewältigung der jüngsten Finanz- und Wirtschaftskrise kam es zu keinen echten Ergebnissen oder gar Entscheidungen, da man die WTO hier nicht als die dafür geeignete und zuständige Institution ansah. Konkret wurde es dann etwa bei Fragen der notwendigen Verlängerung bestehender Regelungen des elektronischen Geschäftsverkehrs und des Geistigen Eigentums. Zum elektronischen Geschäftsverkehr beschloss man, auch künftig keine innerstaatlichen Einfuhrabgaben zu erheben. Ferner verpflichteten sich die Mitglieder, auf dem Gebiet des Geistigen Eigentums von der Möglichkeit, eine Nichtverletzungsbeschwerde (non-violation complaint) ${ }^{6}$ im Rahmen des TRIPS vor dem Dispute Settlement Body zu erheben, weiterhin abzusehen.

\section{Bewertung}

Im Ergebnis ist der Konferenz kein gutes Zeugnis auszustellen. Das Vorhaben, der DohaRunde 2010 in Arbeitskreisen und schon 2011 in einer weiteren Ministerkonferenz endgültig zum Durchbruch zu verhelfen, ist bereits misslungen. Denn bereits während der ersten Konsultationen räumte Generaldirektor Pascal Lamy nach einem Treffen des Generalrats der WTO am 22. Februar 2010 inzwischen ein, dass es für ein weiteres Ministertreffen zu früh sei, da noch zu viele Divergenzen bestünden. Das Ziel der Konferenz bestand wohl eben doch nicht ernsthaft darin, die Doha-Runde zur weiteren Liberalisierung des Welthandels entscheidend voranzubringen oder gar zu einem Abschluss zu führen - ein Unterfangen, an dem die WTO-Mitglieder ja auch in den vergangenen Jahren mehrfach gescheitert sind. Dies bestätigt der Befund, dass in der WTO nach den gemeinsamen Ministerkonferenzen gut gemeinte Vorhaben und verkündete „Ergebnisse“ auf Grund der fehlenden Kompromissbereitschaft einzelner Mitgliedstaaten in der Alltagsarbeit nicht umgesetzt

Vgl. zum Ganzen WT/MIN(09)/18 v. 02.12.2009. Sämtliche offiziellen Dokumente der Konferenz sind im Übrigen abrufbar unter: http://www.wto.org/english/thewto_e/minist_e/min09_e/official_ doc_e.htm.

6 Nach der Nichtverletzungsbeschwerde soll auch ein Verhalten, das ein erzeugtes Vertrauen verletzt, ohne dass eine WTO-Vorschrift verletzt wurde, zur Einleitung eines Streitbeilegungsverfahrens berechtigen, Krajewski, oben Fn. 2, § 2, Rdn. 259. 
werden können. Stattdessen handelt es sich um eine reguläre Konferenz, die sich unter der Überschrift „Die WTO, das Mulitilaterale Handelssystem und das gegenwärtige globale ökonomische Umfeld“ eher nur an der Oberflächliche Probleme behandelt hat, auf die die Minister in ihren Erklärungen Bezug nehmen können. Freilich wird dies in der Öffentlichkeit nach außen hin anders verkauft. Diese Tatsachen bestätigen das erste große Problem der WTO: Endlich Einigkeit zu erzielen um die doch so wichtige Doha-Runde zum Abschluss zu bringen und dadurch eine ausgeglichene Weltwirtschaft und eine angemessene Berücksichtigung der Situation und Interessen der Entwicklungsländer herzustellen.

Das zweite Problem der WTO besteht in ihrem fehlenden Einfluss bei aktuellen Fragen und Krisen, was sich auch wieder bei der letztjährigen Konferenz herauskristallisiert hat. So wurden die Ursachen und Folgen der Finanz- und Wirtschaftskrise angesprochen; zu mehr als einem Appell an die Mitglieder, von protektionistischen Maßnahmen abzusehen, reichte es aber nicht. Vielmehr verwies man die Arbeit an den in der Folge der Krise aufgeworfenen Regulierungsthemen mit dem Argument, dass das WTO-Recht letztlich Marktzugang und nicht für Deregulierung stehe, auf andere Institutionen. Gerade die am wenigsten entwickelten Ländern (LDC) sind jedoch besonders hart von der Finanz- und Wirtschaftskrise betroffen, da deren Exportwirtschaft auf einige wenige Märkte ausgerichtet ist, auf denen ist nunmehr an entsprechender Nachfrage fehlt. Und diese Problematik gehört nun doch eindeutig auch zur WTO-Materie. ${ }^{7}$ Ähnlich verhält es sich bei den Herausforderungen des Bereichs „Handel und Klima“. Die Frage, wie denn die Globalisierung und der liberalisierte Welthandel im Zusammenhang mit dem Klimawandel stehen, wurde in Genf erst gar nicht weiter erörtert. ${ }^{8}$ Bei alldem muss man sich fragen, wieso überhaupt „....das gegenwärtige globale ökonomische Umfeld“ zu einem Thema der Konferenz gemacht wurde. Bei solch fundamentalen Defiziten der Konferenz geht es fast schon unter, dass punktuell bereits bestehende Regelungen, wie die Zollfreiheit bei elektronischem Geschäftsverkehr und die Verpflichtung zum Verzicht der Einleitung von Nichtverletzungsbeschwerden im Bereich des TRPIS, im Wege der formalen Alltagsarbeit verlängert wurden.

Zusammenfassend muss man sagen, dass das Legitimitätsproblem der WTO als supranationale Organisation mit der 7. Ministerkonferenz sich noch verschärft hat. Aber vielleicht ist das, wenn man sich die Konsensfähigkeit innerhalb der WTO vor Augen hält, zur Zeit auch hinnehmbar. Wohlmöglich können andere Akteure und Institutionen zumindest die sich in der Krise akut aufdrängenden Fragen und Herausforderungen der Weltwirtschaft in der Tat zum Teil besser angehen und bewältigen. 2009.

8 WEED-Online v. 02.12.2009, abrufbar unter: http://www.weed-online.org/themen/wto/3105528. html. 